\title{
Engagement in help-seeking of dual diagnosed mothers at a low-threshold service: grasping life through co-created opportunities
}

\author{
Minna A. Sorsa, Irma Kiikkala and Päivi Åstedt-Kurki
}

\begin{abstract}
Minna A. Sorsa is an International co-ordinator at the Department of Child Psychiatry, Pirkanmaa Hospital District, Tampere, Finland; and is at the Faculty of Social Sciences, University of Tampere, Tampere, Finland. Irma Kiikkala is based at the University of Eastern Finland, Kuopio, Finland. Päivi Åstedt-Kurki is based at the Faculty of Social Sciences, Nursing Science, University of Tampere, Tampere, Finland.
\end{abstract}

\begin{abstract}
Purpose - Mothers with a dual diagnosis (mental ill health and substance use) have delays in accessing services, or their care may be interrupted prior to therapeutic relationships being formed. The purpose of this paper is to explore and describe how engagement merges in the context of mothers with a dual diagnosis. Design/methodology/approach - This is a qualitative, focused ethnographic study at a low-threshold service for substance abusing families. The data contain interviews, observations, field notes, and reflections. The analysis followed several systematic steps.

Findings - Engagement is the co-creation of possibilities between workplace staff and the client in different interfaces. It is not a single act, emotion, or verbal communication, but a complex intertwined system of events that can awaken or enable the client to get a grasp on life. The sensitivity of the worker is one tool for engaging the client in manifold ways in different interfaces: even the smallest events with connection are viewed as valuable. Engagement involves the intentional client in the process of interaction: the client needs to participate and become an acting and sensing part of the change, which occurs on an experiential level. The process is described with the metaphor of a seed.

Originality/value - Engagement as inner involvement challenges the current working methods, and requires sensitivity, because the mothers with a dual diagnosis may not have verbalisable goals when arriving to the services.
\end{abstract}

Keywords Engagement, Ethnography, Help-seeking, Dual diagnosis, Mothers/mothering

Paper type Research paper

Mothers with a dual diagnosis (mental ill health and substance use) and vulnerable backgrounds of abuse and experienced violence may have delays in accessing services (Blegen et al., 2010; Tsantefski et al., 2015). As their life problems may be rooted across generations (Wilson et al., 2013), the mothers may feel different, rejected, lonely, and experience exclusion from everyday activities such as relationships and employment (Chorlton and Smith, 2016). The fear of imperfect motherhood and stigma may lead mothers to experience failure, shame, and guilt (Blegen et al., 2010) and powerlessness (Sorsa and Åstedt-Kurki, 2013). The dually diagnosed may be difficult to engage in the services because they may lack hope and appear reluctant (Coombes and Wratten, 2007).

In general, mental ill health and substance use can create delayed help-seeking and may result in negative outcomes with more symptoms, poorer functioning, and less satisfactory quality of life (Clement et al., 2012; McKee, 2017; Nutt et al., 2017). Persons with a dual diagnosis may not know where to look for help, since the services may seem bureaucratic or the system complex, and they may have difficulties in engaging with the services (Coombes and Wratten, 2007; Motta-Ochoa et al., 2017; Ness et al. , 2014; Staiger et al. , 2011; Tsantefski et al. , 2015). People, who do not seek help in questions related to dual diagnosis, do so because of economic constraints, inconvenient opening hours, and unavailability of help (McKee, 2017; 
Motta-Ochoa et al., 2017; Nidecker, et al., 2009). Clients may want to avoid being labelled mentally ill and addict (Motta-Ochoa et al., 2017), and thus mothers may not seek help (Blegen et al., 2010; Sorsa and Åstedt-Kurki, 2013). Women may avoid services for practical reasons due to lacking childcare or on emotional basis, as a fear of their children being taken into custody (Blegen et al., 2010; Tsantefski et al., 2015). However, attendance in services has been recognised as one of the most important predictors of positive outcomes in addiction treatment (Schulte et al., 2010).

Individual staff members may not feel comfortable to connect with clients who are demanding, non-collaborative, or unwilling to participate (Spiers and Wood, 2010). Perhaps due to the complexity and contradictions raised by these clients, it seems that staff consider the dually diagnosed among the most challenging client groups, as staff experience frustration, helplessness, and negativity (Schulte et al., 2010). As the worker's role is emotionally demanding, supporting a positive clinical environment requires training, and experiences of collaboration with this group of clients in order to enhance understanding towards the clients (Nutt et al., 2017). With such complexity and vulnerability of clients, the staff need to put more effort into engaging clients, and use such tools as respect for their clients and belief in the client possibilities (Holm and Severinsson, 2011; Ness et al., 2014; Nutt et al., 2017; Peplau, 1991). Staff need the capacity and willingness to share uncomfortable experiences, so that they can work towards engagement and ongoing therapeutic work (Thibeault, 2016). Whilst therapeutic optimism is important, a positive approach only is not sufficient to prevent dropout. Practitioner's self-rated competency has been linked with a better ability to address and support clients' diverse needs, and thus enhancing engagement (Schulte et al., 2010).

Meeting the client as an individual may give the dually diagnosed a feeling of being valued, which may enhance active engagement (Stanhope, 2012; Ness et al., 2014). Mothers with a dual diagnosis may not seek help, since they may experience shame and failure due to their illness, which may prevent them from disclosing their problems to professionals (Blegen et al., 2010; Chorlton and Smith, 2016). It is, therefore, essential to look for ways of enhancing their commitment and engagement in care. Help-seeking and engagement are connected as terms, since a phase when a person is not yet willing and motivated into treatment require staff to focus on creating a therapeutic relationship (Mueser and Fox, 2002; Prochaska and DiClemente, 1992). The therapeutic approaches should be modified according to the client willingness to be involved (McKee, 2017; Mueser and Fox, 2002; Prochaska and DiClemente, 1992).

In Peplaus's (1991) theory, help-seeking is the phase of orientation, as most clients are ambivalent and may not necessarily be able to express their expectations. The engagement strategies in the care of the dually diagnosed were listed by Mueser and Gingerich (2013) as assertive outreach tools, support on practical questions such as clothing and food, support in the client social network and crisis resolution. Assertive outreach refers to actively reaching to the clients to involve them in treatment (McKee, 2017). Engagement has been linked with both a phase when a client is not connected to care providers, and an already existing therapeutic relationship and involvement of family members (Lizardi and Stanley, 2010). Engagement can mean commitment to using services at a practical level, but it can also mean emotional involvement on an individual level (Stanhope, 2012). The latter is less well known, as there are no existing and comprehensive definitions on the type of help required for inner emotional involvement to appear. A focused ethnographic study at a low-threshold service for substance abusing families was conducted. The aim was to explore and describe how engagement is merging in the context of mothers with dual diagnosis.

\section{Method}

Focused ethnography has evolved as a method to improve care and care processes, and is suitable for studying specified questions, within subcultures in particular settings (Higginbottom et al., 2013; Rashid et al., 2015). The goal of ethnoscience is to systematically collect qualitative data by observations and interviews, and through participation in events of a certain culture 
(Geertz, 1973; McFarland et al., 2012). Previously ethnographers explored culture in a broader context, whereas it can currently deal with specific questions such as experiences of single groups (Higginbottom et al., 2013; Rashid et al., 2015). In this study, we link subjective viewpoints with socially established structures of meaning in the qualitative research process in such a way that the structure created reflects the emic view of the participants (Geertz, 1973). Our approach is descriptive and interpretative, as we search for meaning. The culture shapes everyday life experiences, and thus the ethnographic viewpoint is connected to a phenomenological lifeworld perspective - also in pluralised, complex, or ambivalent situations (Desjarlais and Throop, 2011).

\section{The context of the study}

In focused ethnography, it is of importance to locate participants with in-depth knowledge of the area of interest (Higginbottom et al. , 2013). The data were collected at a low-threshold service for drug-abusing women and families in southern Finland. In the national service delivery context, all pregnant women receive maternity care: the country's 311 municipalities are responsible for providing free health care to expectant mothers and their children (Finnish Municipalities and Regions, 2017). A low-threshold service is an umbrella term for many different types of services for drug abusers (Islam et al., 2013). These services aim at reducing barriers to care by engaging clients in services, where they are willing to meet within an inviting atmosphere. In year 2014, buprenorfine (Subutex) was the biggest problem for those entering substance services in Finland. Of all adults $(n=1,777)$ using substance services in 2014, 42 per cent had a child, and 80 per cent were clients of child protection services (Päihdehuollon huumeasiakkaat, 2014). Dual diagnosis is common among mothers with substance-related problems, since 57 per cent of the women within a psychiatric clinic on addiction had a psychiatric disorder (Strengell et al., 2015).

At the research site, which is within child protection services, most of the clients are parents, who arrive by a referral or an order by a social worker, and the parents face the risk that their children will be taken into custody unless they commit to abstinence and rehabilitation. Most of the clients are between 20 and 30 years old, and most have used drugs for many years. The service is community based and funded by several municipalities in the area. It is a joint undertaking with a substitution clinic and a small family ward. There is also outreach into the community and networking with different agencies in the area.

The field research was planned in advance by arranging several meetings with the management and staff of the low-threshold service. Questions about confidentiality and ethical questions related to working with such a vulnerable population required that a confidentiality agreement was signed by the first author. Since the ethnographic researcher's role as a participant or observer in the ethnographic data collection was a core issue (McFarland et al., 2012), the research was planned so that author could interchange between the roles of participant and observer during the study.

\section{Data}

The data collection in this focused ethnography took place over five weeks on 23 separate days (a total of 149 hours). The data comprise 117 field note entries. All 12 members of the staff at the low-threshold service were interviewed using open-ended interviews questions. These interviews lasted 30-90 minutes, the average being 62 minutes. The 12 staff members interviewed were women, they were aged 34-57 (median age 44), ten were counsellors with different training backgrounds in the health and social services, and several had training in other fields prior to their current work. Two people had no education in the caring professions. Additionally two clients were interviewed on four occasions each.

\section{Data analysis}

An inductive approach in the data collection and qualitative analysis was used. Data analysis commenced during the fieldwork, and continued for the duration of the study (McFarland et al., 2012). QSR NVivo 11 Pro software was used to assist data handling. Transcribed notes and interviews were thematically analysed and the preliminary themes from fieldwork arose: 
connecting, available space and material, and doing. The identified names of the themes and classified subsets were related to each theme. An individual-level analysis and the notes containing observations were compared with the interview data. The themes were developed stepwise by asking different questions from the data so that the emerging understanding could be verified. The last phase included the interpretation and synthesis of findings. The interconnection of different themes appeared as a metaphor to describe the complex phenomena and structure of engagement at an experiential level. A metaphor can conceptualise subjective understanding and reveal the structure of experience (Lakoff and Johnson, 1999).

\section{Ethical questions in the study}

The protocol for the research project was approved by the Ethics Committee at Tampere University Hospital. The research project conforms to the provisions of the Declaration of Helsinki (WMA 1995/2004). The study participants gave their informed consent. To ensure anonymity, the individual respondents are not presented in the citations of this paper.

\section{Results}

The following five themes were identified to explore engagement. The themes we describe are: recognising the context with vulnerability, caring for mothers in human-to-human encounters, connecting through different interfaces: sensitivity as the tool, focusing on the development of the client's wishes and hope in manifold ways: even the smallest events are valuable, and engagement is the co-creation of possibilities at an experiential level - the seed.

\section{Recognising the context with vulnerability}

The professional work necessitated knowledge and a deep appreciation of the background of the clients. The context appeared to be complex, evoking discord, and discrepancies in the workplace: at the same time as the staff were authorities within child protection, they were required to negotiate the difficulties arising from their clients' past. Many clients have experienced childhood neglect, violence, loneliness or rejection, and have missed out on family support:

This led to anxiety and mental health problems, mainly anxiety, which was medicated because there are no tools, no means at home to work with.

Many clients held fears of being assaulted, a background viewpoint leading to mistrust, which may have added to the clients' vulnerable experiences of loneliness. Perhaps their adolescence and development at the time of starting drug use were interrupted and replaced with involvement in the drug community. Being part of a hidden community, characterised by crime and violence, with ordinary, everyday life becoming focused on looking for drugs, finding the means to afford drugs, or obtaining drugs by theft or other criminal behaviour meant the clients drifted into such a lifestyle where the so-called normal things in life were outside the personal sphere:

A 21-year old mother, who had spent 9 years in the drug community had never used electronic bank payments and had no debit card, so even shopping is not simple.

All the clients had experienced emotionally burdensome and insecure years. Many had experiences of relapses and failures in relation to addictive substances and attending services. The workers built a description of their clients as utterly vulnerable, having suffered arduous experiences and lacking the ability to cope with different challenges in life:

The boundaries are tested, since nobody previously set any: the family had no boundaries, and in the drug community no limits exist, since heaven is the only limit.

Staff described times of using and non-using, where the ability of the client changes according to whether they use drugs or are abstinent. Upon arrival at the low-threshold service, the mothers and fathers are tired or even exhausted. Upon entering this service, many of the women are pregnant for the first time or in the early stages of their pregnancy, while others are mothers or fathers of small children. This is a main motivational perspective when the parents seek help themselves. Growing into motherhood and fatherhood were just some of the challenges they had 
to face. Parenthood may have reminded them of their own experienced insecurity. One part of the staff's understanding and commitment to their clients grew out of a deep-rooted understanding and empathy for their context of disruption and vulnerability:

These mothers do not have it: they did not get the chance. They may have started cycling through institutions, and they may have been placed so that they could never express their negative feelings, which fed their addiction even more, so it suppressed the pain and this is why we talk about dually diagnosed clients. It is during their painful times that they should have received proper treatment, but then the drugs came, which helped.

The staff had to deal with disruption and vulnerability. The ambivalent help-seeking needs changed from eagerness to be helped into avoidance of receiving help. Clients described their situation of not identifying a need for help, and they explained that they did not know where or how to look for help. On the contrary, clients quickly changed their mood and help was not accessed:

She cannot ask for help, because it is a sign that she is not coping with her child, so it is a problem.

From the perspective of the staff, their attitude was approving the clients no matter what, they needed the knowledge base and professionalism. These were intertwined so that negotiation and belief could be used to create consistency, trust, direction, and a sense of strength. The staff needed to be deeply involved in their clients' overall outlook on life.

\section{Caring for mothers in human-to-human encounters}

The underlying thinking of the staff was a belief that clients can only be helped with an appreciative and caring approach. This cultural viewpoint was expressed in the workers' approach to care:

If I take really good care of them and treat them well with respect, like ordinary people, I think that they will likewise do the same with their children, so that it can somehow transfer: they receive care and good experiences in a human relationship, and then they manage to transmit this to their children and other relationships.

The staff saw their work and attitude being deeply meaningful also for the children in the families attending. The staff needed various ways of relating with clients, which was visible in everyday events such as how the staff met with clients as they welcomed them. The connections occurred in the many interfaces. The human-to-human approach with empathy and a supportive attitude is not sufficient alone. Perseverance is required to hold on to the client, so that the client would get a better chance of connecting:

We hold on to the client and the most important thing is that we will not let the client vanish. We just

hold on, we keep in contact, and we pick up and we follow the client wherever needed.

Especially at the beginning, it may take several occasions before the staff actually meets with the clients, as the clients do not necessarily arrive as agreed to meetings. This knowledge helps the workers in their attitude towards their clients and their appreciation of the clients' decisions. This requires constant evaluation of how to connect:

We leave a message, we call and say that we are here, you are on our minds, we will call you again tomorrow, we are worried, and how are you?

The staff aimed towards prolonged attunement, holding on to the client, patience and also persevering in situations of ethical difficulty. A professional and appreciative approach is especially complicated for the staff if the client is behaving inappropriately towards a child:

She is enraged, irascible, and yells at the child as I go inside with my colleague. As we have a good relationship, we know how to give feedback in such a way that the mother gets a grip of herself, "hi, what's with you? Consider this from your child's viewpoint, what is your son thinking now and why is he behaving like this?"

The staff must connect with the clients on an emotional level, since knowledge alone does not bring about a caring approach. The cultural order of the workplace requires appreciation and acknowledgement that clients are first of all parents, mothers, fathers, women and men, and only second individuals with substance and/or mental health problems. 


\section{Connecting through different interfaces: sensitivity as the tool}

As the clients can arrive at any time when the service is open, any staff member may encounter them at their arrival. The first moment is considered of importance in forming some type of connection:

If a new client arrives, she comes with a pal or alone, and you as the worker are there from the first moment and welcoming her, so the client connects with you. I think the client connects with the person who meets her. The first contact created can influence the level of trust even further.

The staff see themselves connecting with clients via their presence, which helps in finding the correct way of responding, behaving, and giving feedback to the client. A non-verbal level of communication may be reached via astute listening and conscious choices. Presence is described as being attentive and it requires sensitivity:

My most important task is to be present, to sit there and be present, being available when somebody needs help.

The clients engage in human connections, they do not engage with treatment models, with a site and its services, or via structures, but via normal human habits and steps in connecting with others:

It is quite easy to engage with a human who looks you in the eye and listens to you, and speaking, doing, and gestures discloses that you are considered important and cared for.

The experiential level is where meeting the person (kohtaaminen) occurs. It involves acts, togetherness, and an approach with a supportive attitude. There is a rich setting of small items that may become significant from the client's perspective. If the connection with the client is successful, the client may want to return. Some of the clients arrive at the service while intoxicated. At this low-threshold service, there is an understanding that inevitable relapses occur. For the staff this means that they need to acknowledge the status of clients and the possibility of whether drugs were used that day. At this stage, the clients are not yet fully engaged in collaborating, but the staff and clients are creating models for rehabilitation at the same time. All workers in the team help the clients to develop their grasp of life (tarttumapinta):

It is like a story of recovering from addiction, and it can start whilst being intoxicated. What arises is interest, and they may still continue using [...] engagement occurs before they are drug-free. Yes, I think rehabilitation can start earlier.

The clients have taken major steps prior to accessing services, and this centre is intended for them. The staff do not want to blame their clients; instead, they encourage all clients to arrive on both good and bad days. The workers' attitudes have an impact and they see the complexity also in help-seeking, whether it is expressed openly or not:

We here will not say oh oh, we think the person has herself done a lot of that. The structure was built by starting to think of a place where these people would dare come, even though they have such a severe substance problem, so terrible that somebody might possibly take away their kids.

They recognise that the mothers need to do the mental work in order to make decisions. Arriving at the site can be viewed as an act of courage and concession, since the clients have faced their connection with drugs:

It is a quite hard step to take to enter as a mother and to admit you have an addiction problem, that is tough, and to admit oneself, that yeah I have a problem with spirits, pills, or drugs.

Arriving may mean that the client is labelled as a drug-abusing parent, and even non-using family members have to face this barrier, since it is generally known that this site is intended for families with substance abuse problems. As it is possible to arrive anonymously, some may attend without connecting for a longer time prior to contacting staff:

A mother came here for quite long, and did not talk to staff or anybody, she just came with a mother who had a relationship with us and suddenly BANG, this mother, who had not connected with anybody, said now I want to talk, I have a problem. She had been here for two months just contemplating the situation [...]. And then she dared to ask for help. Then there are also those mothers who already have the problem, they step in and say it aloud.

Many mothers arrive needing help, but they cannot verbalise their need. For the staff, it is not always possible to know how a person can be helped, nor can it be predicted. For the staff to 
create engagement, they need to listen carefully, ask complementary questions, and give supportive feedback. This can occur via the different interfaces, such as action provided at the low-threshold service (Table I).

Staff may use new forms of communication, such as using e-mails and text messages. Their approach needs to be very sensitive, respectful, flexible, changeable, and opportunity dependent. When meeting and connecting with the client, the workers' sensitivity requires an inner endurance: they must be present and listen in a caring manner. They wait for the client to make a wish, even a tiny one. This is the route to creating any movement, action, a glimpse of hope or trust, and eventually engagement. An outsider cannot step in and tell the client how her problems could be solved in the easiest way, since such an approach may be too external or too active, ignore the client's perspective, and may not include the co-creation of wishes. The client decides what help she can accept. If the client senses help being imposed externally and no meeting occurs, she can feel excluded.

\section{Focusing on the development of the client's wishes and hope in manifold ways: even the smallest events are valuable - space and availability}

After a person has entered the facilities, connecting can appear via deeds, in different small moments, notifying someone, and asking to start a discussion. There are many areas or interfaces where action can be taken. There are hints of everyday life, yet this is not everyday life: the focus is on the goal of development of the client's wishes and hope in manifold ways in order to co-create possibilities via space and availability. These interfaces create a culture where connections can occur via human relations or through actions and how things are done. The rooms are designed to invite clients to use the facilities. The space creates a possibility as such for engagement in the rooms and the activities taking place in them.

The biggest room in the service facilities is a large lounge, which contains sofas, toys, and a television; it is a space for passing through to any other room in the building. It contains a big table, which seemed to be the heart of unofficial discussions. A notice board contained the daily schedule and info about available recreational activities. The big sofas in the room invite the clients to relax or take a nap. Even though the staff's work may have appeared to involve hanging around sometimes, in fact they need to be aware of each moment and what followed from any discussions, for example. The counsellors are all individuals and have their personal way of working and approaching the client, many described small micro-moments of communication:

I always consider carefully, should I say 'no', and if I say 'no', why do I say so?

Everybody spends time in the lounge: the clients, family members, and the staff spent time at the table. Lunch was available every day. It seemed as though time stopped at the table:

They come to eat and spend time - quite long time here - at the low threshold service, to have discussions that are a little lighter.

Table I Interfaces through which engagement can be created (identified in field work)

Space and materials

Children's playroom, toys, food table, coffee, food, sofas, possibility to make phone calls (taking care of things, the workers can be reached by cellphone $24 / 7$, opportunity to send SMS messages), using the internet, doing laundry, recycling children's clothes, sauna, recreation: tickets to ice hockey games, movies, access to a gym, aesthetics in decoration Obstacles: the location outside the city requires coming to the service specifically, no space available
Human relations

Actions and practices

Opening the door, meeting a human as person, Location of the site, knowledge of the site via client-worker chemistry: a bridge, smiling, looking/being seen, listening/being heard, other freedom to come and go, wish to return, clients: both known and unknown, different groups, access to childcare, eating together, peers: others in the same life situation, special expertise: discussions, counselling, gynecology services

Obstacles: being bypassed, considered not important, being in a hurry, absence of special expertise network, flyers, internet, One's own place with anonymity, suitable opening hours, meeting each individual, the core attention and interest is on the whole family, a respectful and appreciative attitude, permissiveness, the referring worker may require participation and screenings: the consequences? Obstacles: being bypassed, insufficient worker resources, conflicting schedules, client's life rhythm does not fit opening hours 
Many clients come for lunch, and the large lounge is like an arena for informal and formal peer discussions. Eating is important, but it is not only about the food:

It has somehow transpired that we offer food here. If there was only a smorgasbord, but there were no workers - humans - it would not be nice to come here.

At the specific lunch hours, sizable groups can attend; the adults talk while the children play on the floor. Food and the act of eating together play a major role at the low-threshold service. Food is an entrance to eating traditions, memories, smells, and tastes; it is a basic need and an example of everyday routines. It also serves as an example of eating habits and food preparation. There is a specific positive atmosphere around the table:

The kitchen is at the heart, like the mother's role.

Many staff members sit beside the clients with babies in their lap. The staff find that connecting with the babies is a way of connecting with their clients. The smaller rooms are used for intimate and personal discussions between clients and staff, or for official meetings between different service providers, such as gynecological care. It was possible to wash and dry clothes at the service facilities. Additionally, there is a shower and sauna for the clients' use. The space provided offers many interfaces for connection to occur, if the client wishes so. The staff are aware and recognise that for some clients, accepting help is a necessity for change to occur: these various steps in the process of change are necessary. For some clients, issues may exist only on the non-verbal level. Taking action can be a step without verbalisation, and connecting can occur in a number of ways and in different specific, even detailed situations:

Such things have happened that usually we talk about heavy stuff, and, for example, the colours and fabrics trigger specific memories. By questioning emotions and thoughts, we can go through many things.

The staff can see their clients' grasp of life growing:

Always when an action starts moving from somewhere, it starts to look for its own channels and develops certain things.

In this low-threshold culture, the viewpoint is that no matter how small it may be, a small change may lead to a bigger change in the future, and hope may emerge. Since the mothers have hidden, unrecognised, and complex problems that have an impact on their own lives and those of their children, many ways of helping and connecting are needed. The opportunities and the perspective of hope may increase engagement, since the main motivation of the clients is motherhood:

Pregnancy, yes it is very important to a woman, and in all the mothers I have seen pregnant, they ponder many things, and do so differently. They really think of the baby's well-being [...] but if they get no connection to that they themselves, it can easily occur that after the child is born, 'what does matter anymore?', and substance use can continue. But if they at the same time feel that they themselves are of importance, and they see the significance of staying sober, then there is a longer-lasting impact.

Client engagement may be possible for the time until the baby is born, and it is preceded by meeting the person, the creation of any type of movement.

\section{Engagement is the co-creation of possibilities at an experiential level - the seed}

The themes described above are interconnected; engagement is the co-creation of possibilities at an experiential level. The work entails complexity in the negotiations over vulnerability. The client wishes and hope may awaken as a result of connecting in between the client and staff at the different interfaces (Table I). At a phenomenological level, engagement involves the intentional client in the process of interaction: the client needs to participate and become an acting and sensing part of the change, which occurs on an individual and experiential level.

The cultural model and staff work create inner change within the client, and as the staff described that however small, the change is of value for engagement to occur. The experiential level and the merging engagement is like a seed growing (Figure 1). This is a metaphor that connects the previous themes so that it informs of the goal, and tells about the needed changes, as well as pays a focus on the individual mother as the key actor. The background context of disruption and 


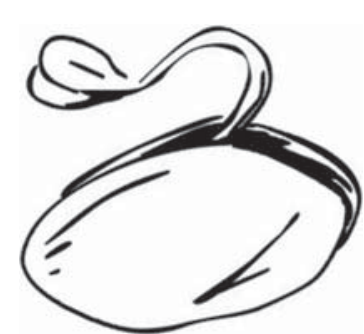

The Metaphor of a Seed

By nurturing a seed, it can sprout and start growing. It does not grow

unattended, or in soil that is too dry

The plant needs to have appropriate soil, light, water and nurturance in such portions that growth can become possible

circumstances is such that growth was not possible prior to accessing the service. The circumstances need to change so that growth and change can become possible. The seed contains itself the knowledge, and the outside conditions are supporting the inner possibility of growth.

The sensitivity of the worker is one tool for engaging the client in manifold ways: even the smallest events with connection are viewed as valuable in order to make the client grasp of life grow (tarttumapinta). It involves a phenomenological experiential level that enhances opportunities for change where ideas, thoughts, and wishes can arise.

\section{Discussion}

The aim of the study was to explore and describe how engagement is merging in the context of mothers with dual diagnosis.

The advantage of an intensive field study is collecting several data sets giving diverse viewpoints of the area of interest. By the time of the analysis, these yield information that support the findings from other types of data. In considering the trustworthiness of the study, a systematic qualitative method was used and the decisions made in the course of the study were described. The fieldwork phase as well as original data were presented in order to ensure credibility. Confirmability was established by comparing the fieldwork observations with the interview data, and going back and forth in data. The interpretation and understanding of actions, events, communications, and other activities within the low-threshold service provide the meaning in context. Using the metaphor was a novel way of approaching the formation of themes. A recurrent pattern in the cultural perspective as a way of working at this specific low-threshold service was found. A limitation of the study is that the study contains mainly staff excerpts from a single site. The client interviews focused on their experiences in a wider perspective. McFarland et al. (2012) include saturation as a criteria for evaluation, and of the usability and richness of the data. The phenomenological lifeworld perspective including the synthesis of findings as a metaphor needs further studies. The research question on engagement is so wide, that a single study does not give answers once and for all.

We identified a complex phenomenon in the field of dual diagnosis: there is a process consisting of smaller steps that leads to the client gaining an ever-greater grasp on life. Engagement is the co-creation of possibilities at an experiential and emotional level.

We identified staff regard towards their clients, a need to accept the background of their clients in order to be supportive and be able to help. The regard of professionals involved in the care of clients with a dual diagnosis has not been well explored, and it may be a component of the stigma and barriers experienced by clients (Nutt et al., 2017). We identified that many clients do not have verbalisable wishes as they arrive, so staff need an encouraging approach, in order to focus on the development of the client's wishes and hope in manifold ways. In the beginning most clients are ambivalent and may not be able to express their wishes (Peplau, 1991). This is in contrast with the service system, which seems to take the client's active participation for granted, and if 
participation is not possible, as for mothers with a dual diagnosis, a negative attitude towards the clients as non-compliant member of society may follow (McKee, 2017; Shrivastava et al., 2012).

In dual diagnosis a person may experience hopelessness, which may be interpreted by others as passivity or unwillingness to be helped, without sufficient knowledge about mental ill health and substance use. In fact, in these situations the clients may be excluded from help. The diagnostic labels in regard to substances may hinder from listening to the unique life stories of clients and may inhibit the listener to understand the lived experience of these clients (Nehls and Sallmann (2005); Coombes and Wratten, 2007). At this site, staff have consciously worked to overcome such a stigma and instead have searched for a stance where listening to the client is central to their way of working. Professionals negotiate their different positions in regard to clients whilst striving at engagement in therapeutic work (Thibeault, 2016). An example is that staff need to overcome such situations as dislike for substances, or anticipation for clients who are unwilling to participate or are ambivalent (Peplau, 1991; Spiers and Wood, 2010). Practitioners' self-rated competency has been linked with a better ability to address and support clients' diverse needs, and thus enhancing engagement (Schulte et al., 2010).

The literature on dual diagnosis focus on expertise knowledge as a requirement (McKee, 2017; Motta-Ochoa, et al., 2017; Nutt et al., 2017; Schulte et al. , 2010). In our study, a tool for engagement was a humane and natural attitude towards the clients. Humaneness and a non-judgemental attitude have been mentioned also in previous studies as tools for engagement to occur (Motta-Ochoa et al., 2017; Nehls and Sallmann, 2005; Ness et al., 2014; Peplau, 1991; Spiers and Wood, 2010; Stanhope, 2012). If a client perceives that she is valued and matters as a human, engagement at an emotional level may be enhanced (Stanhope, 2012). In our data, the staff expressed their will to consider the client first and foremost as a mother with ordinary life questions, and only second as a person with a dual diagnosis, or the stigmatised addict. An approach of humaneness and the everyday life level give the possibility to connect on a level where the professional and client may understand each other's human qualities (Thibeault, 2016).

If mothers arrive to the services with the sense of exclusion and shame, they may have problems in being accepted and building a relationship (Chorlton and Smith, 2016). One step for staff is to rebuild tools for establishing the possibility to engage in relationships. Caring for persons suffering from emotional pain requires staff to expand their own capacity of meaning, so that understanding the other becomes possible (Holm and Severinsson, 2011). Staff need appreciation, caring, and also a long-term viewpoint with perseverance, not giving up, as the mothers with a dual diagnosis will use several occasions in the help-seeking phase prior to engagement. Previously engagement was conceptualised as engagement to treatment or reducing the use of substances (Mueser and Gingerich, 2013). In our study, the staff expressed that these mothers continue the process of engaging on an emotional and inner level also in phases of relapses, which traditionally may have meant dropout of services.

Many of the mothers at the low-threshold service did not have clear wishes, needs and expectations, as they arrived, but engagement meant that these could be co-created with staff within small micro-moments and interfaces. As many clients first try to solve their experienced problems themselves, and use their personal networks, seeking help may be delayed so that early intervention is not possible. The engagement stage, when motivation into treatment does not yet exist, has been described as the precontemplation stage by Prochaska and DiClemente (1992) and Mueser and Fox (2002). It is possible that this stage can be more complex than known previously. Engagement is not only participation in treatment, but also emotional engagement at an individual, emotional and experiential level.

We described the interfaces within human relations, but also in actions and practices, as well as space and materials (Table I). All these areas may create an experiential move within the client, and an interest in something, which is a step into engagement. Interfaces include a non-verbalisable level of actions and the possibility of connection. From a staff viewpoint, being attentive and open in these interfaces requires tools such as sensitivity, respect, and flexibility. Interfaces can enhance co-creating possibilities and movement within the client. As the services have set limits to their availability and the type of services they offer, the interfaces and micro-moments identified in our study would need more research as to clinical practice with mothers with a dual diagnosis. 
Staff meet with the clients vulnerable past in the everyday contacts in the different interfaces (Table I). These are moments, when professionals act so that their attitude becomes known by the client. The examples of interfaces of connection occurring are: space and materials (such as availability of food), human relations (such as meeting a human as person), and actions and practices (such as how the site can be accessed) (Table I). This type of a micro-level and detailed approach with the goal of creating opportunities is not necessarily the aim of dual diagnosis services. Our study shows that very small moments may be a route to "make the seed grow" (as we stated in the metaphor). Brief therapies use the small moments actively with a predesigned model (Spiers and Wood, 2010). The small events can be created in interfaces, as trying to find the common rhythm with the client (Spiers and Wood, 2010).

The perspective of the study gives valuable information about what helps the dually diagnosed mothers: the service needs to use all possible efforts to reach these clients on a humane level so that engagement may follow. The small moments occur in the interfaces and are directed towards the development of client wishes and hope. It can involve a certain way of doing, small moments of communication, availability and presence. The staff noted that no matter how small a change, it may lead to something bigger, and it is always a valuable start, opening new possibilities. This inclusive and appreciative approach is a way to provide easy access to services and engage hard-to-reach clients, a principle following the low-threshold service principles (Islam et al., 2013). The findings of the paper add to the models of good practice that already exist. Further research with a larger number of mothers would be beneficial to test the findings of the study to find out what they find promotes their engagement. Further research could address whether the findings are gender sensitive or applicable also for men.

\section{Conclusion}

Engagement as inner involvement challenges the current working methods, and requires sensitivity, because the mothers with a dual diagnosis may not have verbalisable goals when arriving to the services. Engagement can emerge during the micro-moments, on the many interfaces. Engagement consists of practical issues and commitment, as well as inner involvement. From the staff viewpoint it is not a single act, emotion, or verbal communication, but a complex intertwined system of events that can awaken or enable the client to get a grasp on life. Engagement involves the intentional client in the process of interaction: the client needs to participate and become an acting and sensing part of the change, which occurs on an experiential level. Engagement is co-created by clients and staff.

Further studies on engagement are needed, for example, on the fact that the process of connecting with persons with a dual diagnosis may be much more complicated than described previously. Since the mothers have hidden, unrecognised, and complex problems that have an impact on their own lives and those of their children, many ways of helping and connecting are needed.

\section{References}

Blegen, N., Hummelvoll, J. and Severinsson, E. (2010), "Mothers with mental health problems: a systematic review", Nursing and Health Sciences, Vol. 12 No. 4, pp. 519-28.

Chorlton, E. and Smith, I. (2016), "Understanding how people with mental health difficulties experience substance use", Substance Use \& Misuse, Vol. 51 No. 3, pp. 318-29.

Clement, S., Brohan, E., Jeffery, D., Henderson, C., Hatch, S.L. and Thornicroft, G. (2012), "Development and psychometric properties the barriers to access to care evaluation scale (BACE) related to people with mental ill health", BMC Psychiatry, Vol. 12 No. 36, pp. 1-11.

Coombes, L. and Wratten, A. (2007), "The lived experience of community mental health nurses working with people who have a dual diagnosis: a phenomenological study", Journal of Psychiatric and Mental Health Nursing, Vol. 14 No. 4, pp. 382-392.

Desjarlais, R. and Throop, C.J. (2011), "Phenomenological approaches in anthropology", Annual Review of Anthropology, Vol. 40, August, pp. 87-102. 
Finnish Municipalities and Regions (2017), "Localfinland.fi, Association of Finnish local and regional authorities", 16 February, available at: www.localfinland.fi/expert-services/finnish-municipalities-and-regions (accessed 14 September 2017).

Geertz, C. (1973), The Interpretation of Cultures, Basic Books, New York, NY.

Higginbottom, G., Pillay, J. and Boadu, N. (2013), "Guidance on performing focused ethnographies with an emphasis on healthcare research", The Qualitative Report, Vol. 18 No. 17, pp. 1-16.

Holm, A. and Severinsson, E. (2011), "A hermeneutic approach to the characteristics of mental health nursing practice", Journal of Psychiatric and Mental Health Nursing, Vol. 18 No. 10, pp. 843-50.

Islam, M., Topp, L., Conigrave, K. and Day, C. (2013), "Defining a service for people who use drugs as 'low-threshold': what should be the criteria?", International Journal of Drug Policy, Vol. 24, pp. 220-2.

Lakoff, G. and Johnson, M. (1999), Philosophy in the Flesh: The Embodied Mind and Its Challenge to Western Thought, Basic Books, New York, NY.

Lizardi, D. and Stanley, B. (2010), "Treatment engagement: a neglected aspect in the psychiatric care of suicidal patients”, Psychiatric Services, Vol. 61 No. 12, pp. 1183-91.

McFarland, M., Mixer, S., Wehe-Alamah, H. and Burk, R. (2012), "Ethnonursing: a qualitative research method for studying culturally competent care across disciplines", International Journal of Qualitative Methods, Vol. 11 No. 3, pp. 259-79.

McKee, S.A. (2017), "Concurrent substance use disorders and mental illness: bridging the gap between research and treatment", Canadian Psychology/Psychologie Canadienne, Vol. 58 No. 1, pp. 50-7.

Motta-Ochoa, R., Bertrand, K., Flores-Aranda, J., Patenaude, C., Brunelle, N., Landry, M. and Brochu, S. (2017), "A qualitative study of addiction help-seeking in people with different co-occurring mental disorders and substance use problems", International Journal of Mental Health and Addiction, Vol. 15, pp. 1-17.

Mueser, K. and Fox, L. (2002), "A family intervention program for dual disorders", Community Mental Health Journal, Vol. 38 No. 3, pp. 253-70.

Mueser, K. and Gingerich, S. (2013), "Treatment of co-occurring psychotic and substance use disorders", Social Work in Public Health, Vol. 28 Nos 3-4, pp. 424-39.

Nehls, N. and Sallmann, J. (2005), "Women living with a history of physical and/or sexual abuse, substance use, and mental health problems", Qualitative Health Research, Vol. 15 No. 3, pp. 365-81.

Ness, O., Borg, M. and Davidson, L. (2014), "Facilitators and barriers in dual recovery: a literature review of first-person perspectives", Advances in Dual Diagnosis, Vol. 7 No. 3, pp. 107-17.

Nidecker, M., Bennett, M., Gjonbalaj-Marovic, S., Rachbeisel, J. and Bellack, A. (2009), "Relationships among motivation to change, barriers to care and substance-related consequences in people with dual disorders", Journal of Dual Diagnosis, Vol. 5 Nos 3-4, pp. 375-91.

Nutt, R.A., Gilchrist, G., Marsa Sambola, F. and Baldacchino, A. (2017), "Staff regard towards working with patients who have co-morbid depression and substance misuse: an exploratory study", Heroin Addiction and Related Clinical Problems, Vol. 19 No. 2, pp. 5-16.

Päihdehuollon huumeasiakkaat (2014), “Tilastoraportti 17/2015. Terveyden ja hyvinvoinnin laitos (THL)", available at: www.julkari.fi/bitstream/handle/10024/126907/Tr17.pdf?sequence=4 (accessed 1 January 2018).

Peplau, H. (1991), Interpersonal Relations in Nursing. A Conceptual Frame of Reference for Psychodynamic Nursing, Springer Publishing Company, New York, NY.

Prochaska, J.O. and DiClemente, C.C. (1992), "Transtheoretical therapy: toward a more integrative model of change", Psychotherapy, Theory, Research and Practice, Vol. 19 No. 3, pp. 276-88.

Rashid, M., Caine, V. and Goez, H. (2015), "The encounters and challenges of ethnography as a methodology in health research", International Journal of Qualitative Methods, pp. 1-16.

Schulte, S.J., Meier, P.S., Stirling, J. and Berry, M. (2010), "Dual diagnosis competency among addiction treatment staff: training levels, training needs and the link to retention", European Addiction Research, Vol. 16 No. 2, pp. 78-84.

Shrivastava, A., Johnston, M. and Bureau, Y. (2012), "Stigma of mental illness-2: non-compliance and intervention", Mens Sana Monographs, Vol. 10 No. 1, pp. 85-97. 
Sorsa, M. and Åstedt-Kurki, P. (2013), "Lived experiences in help-seeking from the perspective of a mother with a dual diagnosis", International Journal of Qualitative Studies on Health and Well-being, Vol. 8, pp. 1-12, available at: http://dx.doi.org/10.3402/qhw.v8i0.20316

Spiers, J. and Wood, A. (2010), "Building a therapeutic alliance in brief therapy: the experience of community mental health nurses”, Archives of Psychiatric Nursing, Vol. 24 No. 6, pp. 373-86.

Staiger, P.K., Thomas, A.C., Ricciardelli, L.A., McCabe, M.P., Cross, W. and Young, G. (2011), "Improving services for individuals with a dual diagnosis: a qualitative study reporting on the views of service users", Addiction Research and Theory, Vol. 19 No. 1, pp. 47-55.

Stanhope, V. (2012), "The ties that bind: using ethnographic methods to understand service engagement", Qualitative Social Work, Vol. 11 No. 4, pp. 412-30.

Strengell, P., Väisänen, I., Luukkala, T. and Seppä, K. (2015), "Psychiatric comorbidity among substance misusing mothers", Nordic Journal of Psychiatry, Vol. 69 No. 4, pp. 315-21.

Thibeault, C. (2016), "An interpretation of nurse-patient relationships in Inpatient psychiatry: understanding the mindful approach”, Global Qualitative Nursing Research, Vol. 3, March, pp. 1-10.

Tsantefski, M., Jackson, A. and Humphreys, C. (2015), "A delicate balance: intervention with mothers with dual diagnosis and their infants", Advances in Dual Diagnosis, Vol. 8 No. 2, pp. 78-89.

Wilson, C., Bennett, M. and Bellack, A. (2013), "Impact of family history in persons with dual diagnosis", Journal of Dual Diagnosis, Vol. 9 No. 1, pp. 30-8.

WMA (1995/2004), "Declaration of Helsinki", available at: www.wma.net/wp-content/uploads/2016/11/DoHOct2004.pdf (accessed 3 December 2016).

\section{Further reading}

Staiger, P.K., Long, C., McCabe, M. and Ricciardelli, L. (2008), "Defining dual diagnosis: a qualitative study of the views of health care workers", Mental Health and Substance Use: Dual Diagnosis, Vol. 1 No. 3, pp. 194-204.

\section{Corresponding author}

Minna A. Sorsa can be contacted at: minna.sorsa@staff.uta.fi

For instructions on how to order reprints of this article, please visit our website: 